\title{
Quality improvement strategies for organizational change: a multiphase observational study to increase insight into nonparticipating organizations
}

Jeanny J. A. de Groot ${ }^{1,2^{*}}$ D, Maite Timmermans ${ }^{2,3}$, José M. C. Maessen ${ }^{1,4}$, Bjorn Winkens ${ }^{5}$, Carmen D. Dirksen 6 , Brigitte F. M. Slangen ${ }^{2,7}$ and Trudy van der Weijden ${ }^{1}$

\begin{abstract}
Background: The scope of implementation research is often restricted to the analysis of organizations that participate voluntarily in implementation interventions. The recruitment of participants for a quality improvement collaborative increases awareness of the specific innovation. The objective of this multiphase observational study was to identify differences between organizations that participated in a large-scale implementation project aiming to improve perioperative care, functional recovery, and length of hospital stay after gynecologic surgery and organizations that did not participate. A secondary objective was to explore how perioperative practice changed among nonparticipants.
\end{abstract}

Methods: Of the seven gynecology departments of nonparticipating Dutch hospitals, five agreed to participate in a retrospective analysis. Baseline data of participating hospitals' $(N=19)$ characteristics, time to functional recovery, and length of hospital stay were compared. Outcome measures for the subsequent pre-post awareness study in the five nonparticipating hospitals were: (1) overall adherence to predefined evidence-based perioperative elements; and (2) change in functional recovery and length of hospital stay. Multivariable regression models, adjusted for baseline characteristics, were used for analysis.

Results: In retrospect, nonparticipating and participating hospitals did not differ in baseline characteristics, functional recovery, and length of hospital stay. In three of the five nonparticipating hospitals, adherence to the selected evidence-based perioperative elements increased significantly after awareness of the trial (overall mean difference $9.7 \%, 95 \%$ Cl 6.9 to $12.5 \%, p<0.001$ ). Linear regression models revealed no statistically significant or clinically relevant differences in time to functional recovery (mean difference -0.2 days, $95 \% \mathrm{Cl}-0.7$ to $0.2, p=0.319$ ) or length of hospital stay (mean difference -0.4 days, $95 \% \mathrm{Cl}-1.3$ to $0.5, p=0.419$ ) in the nonparticipating hospitals. None of these hospitals managed to reduce time to functional recovery or length of hospital stay significantly.

\footnotetext{
* Correspondence: jeanny.de.groot@mumc.nl

${ }^{1}$ Department of Family Medicine, CAPHRI, School for Public Health and Primary Care, Maastricht University, P.O. Box 616, 6200, MD, Maastricht, The Netherlands

${ }^{2}$ Department of Obstetrics and Gynaecology, Maastricht University Medical

Centre, P.O. Box 5800, 6202, AZ, Maastricht, The Netherlands

Full list of author information is available at the end of the article
}

(c) The Author(s). 2018 Open Access This article is distributed under the terms of the Creative Commons Attribution 4.0 International License (http://creativecommons.org/licenses/by/4.0/), which permits unrestricted use, distribution, and reproduction in any medium, provided you give appropriate credit to the original author(s) and the source, provide a link to the Creative Commons license, and indicate if changes were made. The Creative Commons Public Domain Dedication waiver (http://creativecommons.org/publicdomain/zero/1.0/) applies to the data made available in this article, unless otherwise stated. 
(Continued from previous page)

Conclusions: No differences in perioperative outcomes between the nonparticipating and participating hospitals were identified at baseline. Despite the statistically significant improvement in overall evidence-based perioperative care, the awareness raised by recruitment activities alone was not enough to reduce time to functional recovery and length of hospital stay in nonparticipating hospitals. Insight into the trends of nonparticipants is valuable to existing implementation effectiveness research.

Keywords: Implementation, Nonparticipant analysis, Awareness, Length of stay, Perioperative care

\section{Background}

Several single and multifaceted implementation interventions have been used to improve the translation of knowledge and guidelines into medical practice [1-3]. These implementation interventions are specified activities designed to enhance adoption of clinical practice with the goal to improve healthcare outcomes [4, 5]. Their effectiveness in optimizing patient outcomes and healthcare quality has been addressed across different contexts, but further evaluations are still required in order to explore the practical implications of different strategies $[2,3,6]$. Whether beneficial implementation effects on health outcome or quality measures will occur and to what extent is difficult to predict in advance [2, 3, 6]. Moreover, the process of change requires the investment of considerable financial and personal resources [7, 8]. These considerations have the potential to influence health organizations' choices regarding the execution of implementation interventions $[2,7]$.

Grol [9, 10] described several specific steps and related barriers involved in the process of change. Orientation, insight, and acceptance are necessary before actual change can begin in practice [10]. Studies have shown that organizational readiness facilitates the successful implementation of innovations and new guidelines [11]. In their review article, Weiner and colleagues conclude that both motivation and capability are essential components of readiness for change [11]. There has been increased awareness of the role of such contextual factors on the effectiveness of implementation interventions in healthcare $[12,13]$.

Achieving large-scale implementation of innovations and new guidelines across intra-organizational boundaries is even more complex. Quality improvement collaboratives are designed to improve healthcare quality and outcomes among multiple organizations by using a structured, temporary approach $[6,14]$. Sponsorship and the type and priority of the specific issue might determine whether organizations participate in those collaboratives [15]. Participants usually take part in quality improvement collaboratives voluntarily in order to improve quality in healthcare [15]. This depends primarily on their willingness to invest and take part. The scope of implementation research is often restricted to the analysis of participants. Specific recruitment processes remain unexamined and could affect the representativeness of the study sample. Although previous studies have addressed nonparticipation in other settings, the evaluation of sample representativeness is particularly interesting in implementation research. In every process of change, target groups can be divided into several categories according to Rogers's diffusion theory [16]. The process by which an innovation spreads among members of a social system over time is described in this conceptual theory. So, it can be assumed that participants of large-scale implementation strategies differ from nonparticipants. Analysis of demographic variables and reasons for nonparticipation could reveal subsequent participation bias.

In Grol's ten-stage model [10], awareness is pointed out as the first orienting step in the process of change. This action model for effective implementation of change formulates a systematic step-wise proposal for change and can be used to plan and support an implementation process. The recruitment of participants in a quality improvement collaborative increases awareness of the specific innovation. We define awareness as a state of knowing and being informed about a clinical intervention to improve health care outcomes. It is assumed that awareness alone usually is not sufficient to change practice [17]. Evaluating the influence of this awareness in terms of how it effects change in medical practice among the nonparticipant group might further understandings of implementation processes. In the present study, we focused on the nonparticipants of the 'Stepped Implementation of Enhanced Recovery in Gynecology' (SINERGY) trial. The SINERGY trial is a nationwide comparative effectiveness implementation study that aims to compare two different large-scale implementation strategies across Dutch hospitals in order to define the most efficient strategy to improve practice [18]. A traditional breakthrough strategy with generic, short-term cyclic educational sessions is compared with an innovative stepped implementation strategy comprising different levels of intensity of support adapted to the specific needs of an organization. Reviews have indicated that implementing evidence-based perioperative elements in gynecologic surgery results in improved perioperative outcomes [19-21].

There were two primary objectives of the current study: (1) to identify differences between the nonparticipating 
and participating hospitals of the SINERGY trial; (2) to explore whether awareness of the start of a perioperative improvement program in other hospitals affected perioperative outcomes in hospitals that did not participate in the improvement program. The overall group effect on outcome measures and differences in effect within individual hospitals will be evaluated.

\section{Methods}

\section{Design}

A multiphase observational study was conducted sequentially. To assess the representativeness of the potentially eligible sample of the SINERGY trial, a retrospective observational multicenter approach was used, which compared nonparticipating hospitals with hospitals participating in the SINERGY trial. Subsequently, a pre-post study was conducted in the nonparticipating hospitals in order to identify any changes in perioperative practice, length of functional recovery, and length of postoperative hospital stay. This study was financially supported by the Netherlands Organization for Health Research and Development (ZonMw) (project number: 837003002).

\section{Setting}

The current study can be seen as an addition to the nationwide SINERGY trial [18]. All the gynecology departments of the 27 hospitals authorized to perform major gynecologic oncologic surgery in the Netherlands were assessed for eligibility. In line with the SINERGY trial protocol [18], one hospital that referred most of its patients to inpatient rehabilitation facilities after hospital discharge was excluded a priori. In total, 26 eligible hospitals were invited to participate in the SINERGY trial.

\section{Recruitment process}

A combination of targeted recruitment efforts were undertaken to increase involvement in the SINERGY trial. In mid-2013 local stakeholders were identified and contacted directly by members of the expert team. A personal letter was sent, which contained information about the study project, the implementation strategies being tested, the objectives of the perioperative guidelines, the need for active implementation, and the expected time and personal commitment required. In conformance with the SINERGY trial protocol [18], and in order to minimize implementation effects before the start of the study project, detailed information about the specific elements of the perioperative guidelines were not disseminated. Local stakeholders were, however, aware of the intentions of the SINERGY trial to reduce time to recovery and length of hospital stay by implementing evidence-based perioperative elements according to the enhanced recovery after surgery (ERAS) approach [18]. Reminders by email and telephone were used consecutively, when necessary. Visits to local organizations to provide additional information were undertaken on request. The study was supported by the Dutch Gynecology Oncology Group (DGOG), which comprises professionals who collaborate to promote research in gynecologic cancer. Funding provided by ZonMw helped to tackle financial disincentives by confining the local costs of implementation to attending meetings and executing local activities. Local stakeholders continued to be approached until a statement of commitment or nonparticipation was provided, resulting in a response rate of $100 \%$. Even though all hospitals acknowledged the need for the nationwide standardization of perioperative practice consistent with ERAS, some decided not to participate in the SINERGY trial (nonparticipation rate $27 \%$ ). These hospitals did not undergo a baseline audit of perioperative practice to determine if they were eligible for inclusion in the SINERGY trial. Stakeholders were asked about their reasons for nonparticipation. A combination of the compulsory nature of collective multidisciplinary meetings, a lack of time and resources due to high workload, and the lack of financial incentives were reported. In addition, nonparticipants questioned the return on their investment of implementing the evidence-based perioperative elements in improving time to recovery and length of hospital stay.

\section{Study groups}

In total, seven hospitals chose not to take part in the SINERGY trial. Five of these seven hospitals agreed to participate in the current study. These 'nonparticipating in SINERGY trial' hospitals were compared with eligible participants of the SINERGY trial in order to address the first research objective of determining sample representativeness. The participant group consisted of hospitals that intended to participate in the SINERGY project $(N=19)$ [18]. For the second research objective, only the nonparticipating hospitals were analyzed for change in perioperative practice and outcomes following recruitment activities for the SINERGY trial. The comparison with participating hospitals will be made in future research, which will report data of the participating hospitals during the implementation interventions in more detail as well. Patients treated in the period before the recruitment process began, and thus before awareness of the SINERGY trial (pre-awareness group), were compared with patients treated one year after awareness of the trial (post-awareness group). This one-year period was chosen because the implementation interventions for the SINERGY trial were to begin in June 2014 [18]. In this way the effect of awareness was isolated as much as possible. 


\section{Outcomes}

Nonparticipating and participating hospitals were compared to identify differences in baseline characteristics before the onset of the recruitment process. The total number of beds per hospital, the type of hospital (university or non-university), and the level of specialized health care (secondary or tertiary) were obtained. Effect measures of the implementation of evidence-based perioperative elements were time to functional recovery and length of postoperative hospital stay. The outcomes in these effect measures were assessed in retrospect. Functional recovery was achieved when patients tolerated a normal diet, mobilized independently, and were comfortable on oral analgesia. Day zero refers to the day of surgery.

The outcomes of the second research objective, to explore the effect of awareness, were overall adherence rate to selected evidence-based perioperative care elements, time to functional recovery, and length of postoperative hospital stay in days. The selection of elements was in line with the SINERGY trial protocol [18] and an overall mean adherence rate was calculated. Selected elements included preadmission counseling and education, no bowel lavage, oral carbohydrate loading before surgery, routine use of prophylactic anti-emetics, nasogastric tube removal after surgery, no use of peritoneal drains, oral fluid intake on day zero, start solid food on the first postoperative day, mobilization on chair at least three times on the first postoperative day, use of epidural analgesia, routine use of oral laxatives, and urinary catheter removal before the third postoperative day. These predefined elements can be retrieved reliably and have a direct influence on recovery. In combination they should reflect an enhanced recovery approach.

\section{Data collection}

In each nonparticipant hospital, retrospective data of two independent, consecutive samples of 30 patients were collected for the pre-awareness (January 2012) and post-awareness (June 2014) group. Baseline characteristics, surgical details, and outcome measures were retrieved from medical records. Patients underwent explorative laparotomies, surgical staging, or cytoreductive procedures for suspected, primary, or recurrent gynecologic malignancies. By selecting this specific group of patients, a comparable case-mix was provided for all hospitals. In addition, hospital characteristics were collected. For the participant group the baseline audit included a consecutive sample of 30 patients treated in 2012. No exclusion criteria were applied at the patient level.

\section{Data analysis}

Absolute numbers with percentages were used for categorical data, while means with standard deviations (SD) or medians with ranges, where appropriate, were used for numerical variables. Differences were assessed using the independent-samples t-test or the Mann-Whitney U test for numerical variables, and the chi-square test or Fisher's exact test, where appropriate, for categorical ones. Both unadjusted and adjusted statistical methods were used to identify differences in implementation endpoints between the nonparticipating and participating hospitals and to assess the overall effect of awareness on perioperative management and outcomes after elective gynecologic surgery. Linear multilevel analysis methods were used to compare the time to functional recovery and the length of hospital stay between the nonparticipating and participating hospitals. Models were adjusted for clustering (random effect) and patient and surgical characteristics (fixed effects). Study group, age, American Society of Anesthesiologists' classification, (suspected) diagnosis and type of surgery, histological subtype, surgical approach, operation time, type of incision, and type of additional surgical procedure were included in the models as fixed effects. To evaluate the overall effect of awareness, the hospitals included in the nonparticipant study could not be treated as a random sample, but were included in the model as a fixed factor. So, multivariable linear regression models, adjusted for patient and surgical characteristics, were used. Furthermore, we were also interested in the separate outcomes for each hospital. Therefore, subsequent analyses of interaction terms between groups (pre-awareness versus post-awareness) and hospitals were performed to determine separate effects. Adjusted mean differences (MD) and 95\% confidence intervals (CI) for results in linear regression models were reported. Multiple imputations (20 imputed datasets) were used to replace missing data on covariates, where appropriate. Since there was only one case with a missing covariate in both the pre-awareness and post-awareness group, complete case analysis was used for the regression models of nonparticipants. In a sensitivity analysis, logarithmic transformation of the data on time to functional recovery and length of postoperative hospital stay was used to analyze the influence of right-skewness of data in the nonparticipant analysis. A $p$-value $\leq 0.05$ was considered statistically significant. A more than one day reduction in time to functional recovery and length of hospital stay (pre-awareness versus post-awareness) were considered clinically relevant. Analyses were performed using IBM SPSS Statistics for Windows, version 21.0 (Armonk, NY, USA).

\section{Results}

Nonparticipating versus participating hospitals

A total of 24 hospitals were compared, five nonparticipating and 19 participating. Although the nonparticipating hospitals tended to be smaller, no significant differences in the baseline characteristics of the nonparticipating and participating hospitals were found (Table 1). Unadjusted 
analysis demonstrated no significant differences between hospital groups in the SINERGY trial effect outcomes, that is, in time to functional recovery $(p=0.205)$ and length of postoperative hospital stay $(p=0.659)$ at baseline (Table 1). Linear multilevel analysis of time to functional recovery demonstrated an adjusted mean difference of 0.3 (95\% CI -0.2 to 0.8$)$ days $(p=0.256)$ between the nonparticipating and the participating hospitals. The adjusted mean difference in length of postoperative hospital stay was 0.0 (95\% CI -0.8 to 0.8$)$ days $(p=0.977)$.

\section{Pre-awareness versus post-awareness group}

In the nonparticipating hospitals, the medical records of a total of 300 patients who underwent elective gynecologic surgery were collected for the pre-awareness $(n=$ $150)$ and post-awareness $(n=150)$ groups. Patient characteristics and surgical details for the patients treated at baseline (pre-awareness) and post-awareness are shown in Table 2. Characteristics were similar in terms of American Society of Anesthesiologists' classification, (suspected) diagnosis, and histological subtype; however, mean $( \pm$ SD) patient age was significantly higher in the post-awareness group $(61.8 \pm 12.2$ versus $65.1 \pm 12.7$ years, $p=0.024)$. Surgical approach, length of surgery, and the presence of additional procedures did not differ statistically between the study groups. More patients in the post-awareness group underwent primary cytoreductive surgery for ovarian cancer rather than an explorative or staging procedure compared to the pre-awareness group $(p=0.027)$.

Adherence rates to the perioperative elements of the ERAS pathway pre- and post-awareness are shown in Table 3. Anti-emetics were routinely used during surgery in $61.6 \%(n=90)$ of patients in the pre-awareness group compared with $74.7 \% \quad(n=112)$ of patients in the post-awareness group $(p=0.016)$. In addition, a significant increase was observed in adherence rates for the avoidance of intraperitoneal drains $(69.3 \%$ versus $81.9 \%, p=$ $0.011)$, early oral intake of fluids $(24.7 \%$ versus $42.6 \%, p=$ 0.001 ) and solid food (55.3\% versus $77.7 \%, p<0.001)$, and early mobilization on postoperative day one (14.1 versus $27.3 \%, p=0.005)$. On the other hand, slightly more patients were pretreated with oral bowel lavage solutions $(0.0 \%$ versus $5.4 \%, p=0.003)$. Overall, there was a significant difference in unadjusted adherence rates to the 12 predefined perioperative elements between the pre- and post-awareness group (mean rate $46.1 \pm 15.5$ versus 53.3 $\pm 13.3, p<0.001$ ) (Table 4). Multivariable linear regression, adjusted for baseline characteristics and surgical approach, demonstrated a similar result (MD 9.7, 95\% CI 6.9 to $12.5 \%, p<0.001)$. A significant increase in the adjusted mean difference in adherence rate was observed for three out of five hospitals (Hospital 2: $p<0.001$, Hospital 3: $p=$ 0.001, and Hospital 5: $p<0.001$ ) (Fig. 1). There was no significant difference in adherence rates to the predefined perioperative elements between the two study groups for Hospital $1(p=0.921)$ and Hospital $4(p=0.875)$ (Fig. 1).

Functional recovery data were obtained for 294 patients (98.0\%). In the pre-awareness group three patients died during admission (2.0\%). In the post-awareness group one patient died shortly after discharge $(0.7 \%)(p$ $=0.622)$. The mean $( \pm S D)$ time to functional recovery was $4.8 \pm 2.4$ days in the pre-awareness and $4.9 \pm 2.2$ days in the post-awareness group $(p=0.892)$ (Table 4). Adjusted analysis of time to functional recovery demonstrated a non-significant reduction of 0.2 days $(95 \% \mathrm{CI}$ -0.7 to $0.2, p=0.319$ ) (Table 4). The effect of awareness on time to functional recovery did not differ significantly for any hospital (Fig. 2).

No statistically significant or clinically relevant difference in the unadjusted mean length of hospital stay after surgery was observed between the pre- and post-awareness group

Table 1 Baseline characteristics of participating and nonparticipating hospitals to the SINERGY trial $(N=24)$

\begin{tabular}{|c|c|c|c|}
\hline & $\begin{array}{l}\text { Participating hospitals } \\
(N=19)\end{array}$ & $\begin{array}{l}\text { Nonparticipating hospitals } \\
(N=5)\end{array}$ & $p$ value \\
\hline Total number of hospital beds, median [range] & 715 [367-1320] & 653 [180-976] & $0.455^{\mathrm{a}}$ \\
\hline Type of hospital, N (\%) & & & $>0.999^{b}$ \\
\hline University medical center & $6(31.6)$ & $1(20.0)$ & \\
\hline Non university teaching hospital & $13(68.4)$ & $4(80.0)$ & \\
\hline Level of care, N (\%) & & & $0.631^{b}$ \\
\hline Secondary care & $12(63.2)$ & $4(80.0)$ & \\
\hline Tertiary care & $7(36.8)$ & $1(20.0)$ & \\
\hline \multicolumn{4}{|l|}{ Time to functional recovery (days) } \\
\hline Unadjusted, median [range] & $4.0[3.0-5.0]$ & $5.0[3.0-6.0]$ & $0.205^{\mathrm{a}}$ \\
\hline \multicolumn{4}{|l|}{ Length of postoperative hospital stay (days) } \\
\hline Unadjusted, median [range] & $6.0[4.0-10.5]$ & $5.0[3.0-7.0]$ & $0.659^{\mathrm{a}}$ \\
\hline
\end{tabular}

Group differences were tested using the Mann-Whitney $\mathrm{U}$ test ${ }^{\mathrm{a}}$ or Fisher's exact test ${ }^{\mathrm{b}}$ at hospital level. $\mathrm{N}=$ number of hospitals 
Table 2 Baseline characteristics and surgical details of the pre-awareness and post-awareness groups

\begin{tabular}{|c|c|c|c|}
\hline & $\begin{array}{l}\text { Pre-awareness } \\
(n=150)\end{array}$ & $\begin{array}{l}\text { Post-awareness } \\
(n=150)\end{array}$ & $p$ value \\
\hline Age in years, mean $\pm S D$ & $61.8 \pm 12.2$ & $65.1 \pm 12.7$ & $0.024^{\mathrm{a}}$ \\
\hline ASA classification, n (\%) & & & $0.757^{b}$ \\
\hline$|/| \mid$ & $124(82.7)$ & $126(84.0)$ & \\
\hline III/IV & $26(17.3)$ & $24(16.0)$ & \\
\hline (Suspected) diagnosis, n (\%) & & & $0.110^{\mathrm{b}}$ \\
\hline Ovarian cancer & $115(76.7)$ & $114(76.0)$ & \\
\hline Uterine cancer & $27(18.0)$ & $34(22.7)$ & \\
\hline Cervical cancer & $8(5.3)$ & $2(1.3)$ & \\
\hline Histological subtype, n (\%) & & & $0.111^{\mathrm{b}}$ \\
\hline Benign & $37(24.7)$ & $27(18.0)$ & \\
\hline Borderline / hyperplasia & $14(9.3)$ & $8(5.3)$ & \\
\hline Malignant & $99(66.0)$ & $115(76.7)$ & \\
\hline Surgical approach, n (\%) & & & $>0.999^{c}$ \\
\hline Midline incision & $147(98.0)$ & $147(98.0)$ & \\
\hline Transverse incision & $3(2.0)$ & $3(2.0)$ & \\
\hline Operation time in minutes, mean $\pm S D$ & $138.9 \pm 72.0$ & $142.7 \pm 67.5$ & $0.632^{\mathrm{a}}$ \\
\hline Type of surgery (only ovarian included), n (\%) & & & $0.027^{b}$ \\
\hline Explorative & $50(43.5)$ & $40(35.1)$ & \\
\hline Staging & $13(11.3)$ & $6(5.3)$ & \\
\hline Primary cytoreductive & $11(9.6)$ & $25(21.9)$ & \\
\hline Secondary cytoreductive & $41(35.7)$ & $43(37.7)$ & \\
\hline \multicolumn{4}{|l|}{ Additional surgical procedure, n (\%) } \\
\hline Open-close (inoperable) & $1(0.7)$ & $5(3.3)$ & $0.214^{c}$ \\
\hline Anastomosis & $6(4.0)$ & $7(4.7)$ & $0.777^{b}$ \\
\hline Diverting stoma & $6(4.0)$ & $9(6.0)$ & $0.427^{b}$ \\
\hline Lymphadenectomy & $34(22.7)$ & $27(18.0)$ & $0.315^{b}$ \\
\hline Extensive upper abdominal surgery * & $4(2.7)$ & $8(5.3)$ & $0.239^{b}$ \\
\hline
\end{tabular}

Group differences were tested using the independent samples t-test ${ }^{a}$, Chi-square test ${ }^{b}$, or Fisher's exact test ${ }^{c}$. ASA $=$ American Society of Anesthesiologists, $n=$ number of patients, SD = standard deviation. ${ }^{*}$ Includes diaphragmatic stripping, partial liver resection, or splenectomy

$(6.4 \pm 4.2$ versus $6.5 \pm 4.1$ days, $p=0.874)$ (Table 3$)$. Multivariable linear regression showed that none of the hospitals had a statistically significant change in the length of postoperative hospital stay (Fig. 3). The overall adjusted mean difference remained non-significant (MD - 0.4 days, 95\% CI -1.3 to $0.5, p=0.419$ ) (Table 4 ).

\section{Discussion}

In this study, we had the opportunity to illustrate the characteristics of and changes in organizations that did not participate in a nationwide comparative effectiveness implementation trial to implement evidence-based perioperative guidelines across gynecology departments in the Netherlands [18]. The findings provide insight into the effect that awareness of the start of an implementation program can have on the perioperative outcomes of hospitals not participating in the improvement program.
The current study found a significant change in overall perioperative practice, resulting in an estimated mean adherence of $64.0 \%$ (95\% CI 51.2 to $76.9 \%$ ) to the selected evidence-based elements in the post-awareness group. Analysis of the individual perioperative elements showed that more routine anti-emetics were administered and less intraperitoneal drains were used during surgery. In addition, an increase in early oral intake and early mobilization rates was found. Nevertheless, no statistically significant or clinically meaningful reduction in time to functional recovery or length of postoperative hospital stay occurred in the post-awareness group (MD 0.2 days, $p=0.319$ and $\mathrm{MD}-0.4$ days, $p=0.419$, respectively). These results support previous research findings regarding guidelines for enhanced recovery after surgery, which indicate a $70 \%$ cutoff as the minimum level of adequate adherence $[22,23]$. The enhanced recovery after 
Table 3 Adherence rates to the selected perioperative care elements

\begin{tabular}{|c|c|c|c|}
\hline & $\begin{array}{l}\text { Pre-awareness } \\
(n=150)\end{array}$ & $\begin{array}{l}\text { Post-awareness } \\
(n=150)\end{array}$ & $p$ value \\
\hline \multicolumn{4}{|l|}{ Preoperative items, n (\%) } \\
\hline Counseling and education & $0(0)$ & $0(0)$ & \\
\hline No bowel lavage & $150(100)$ & $140(94.6)$ & $0.003^{b}$ \\
\hline Oral carbohydrate loading (if indicated) & $32(21.3)$ & $35(23.3)$ & 0.782 \\
\hline \multicolumn{4}{|l|}{ Intraoperative items, n (\%) } \\
\hline Epidural analgesia & $121(80.7)$ & $126(84.0)$ & 0.451 \\
\hline Routine anti-emetics & $90(61.6)$ & $112(74.7)$ & 0.016 \\
\hline No use of drains & $104(69.3)$ & $122(81.9)$ & 0.011 \\
\hline Nasogastric tube removal & $105(70.0)$ & $112(75.7)$ & 0.272 \\
\hline \multicolumn{4}{|l|}{ Postoperative items, n (\%) } \\
\hline \multicolumn{4}{|l|}{ Avoidance of ileus } \\
\hline Oral laxatives at day 1 & $33(22.0)$ & $42(28.0)$ & 0.232 \\
\hline \multicolumn{4}{|l|}{ Early oral intake } \\
\hline Oral fluids intake day 0 & $37(24.7)$ & $63(42.6)$ & 0.001 \\
\hline Solid food intake $\leq$ day 1 & $83(55.3)$ & $115(77.7)$ & $<0.001$ \\
\hline \multicolumn{4}{|l|}{ Early mobilization } \\
\hline On chair (minimum 3 times) $\leq$ day 1 & $21(14.1)$ & $41(27.3)$ & 0.005 \\
\hline \multicolumn{4}{|l|}{ Early removal of catheters } \\
\hline CAD removal $\leq$ day 2 & $51(34.2)$ & $47(31.5)$ & 0.623 \\
\hline
\end{tabular}

surgery guidelines developed by the ERAS Society include over 20 elements that, according to the best evidence available, are necessary for optimal perioperative care [24, 25]. The guidelines are used to reduce the gap between research and practice and aim to minimize the stress response to surgery [26]. As a result, functional recovery and time to discharge are supposed to improve. In accordance with the SINERGY trial protocol [18], we analyzed adherence to 12 of these perioperative elements. These predefined elements can be retrieved reliably and have a direct influence on recovery. In combination they should reflect an enhanced recovery approach. The elements were equally weighted to calculate the adherence rate as a measure of the implementation process. Perioperative data were obtained retrospectively through the review of medical records, which may have introduced bias to the results.

On a hospital level, three out of five hospitals achieved a significant improvement in evidence-based perioperative

Table 4 Unadjusted and adjusted outcomes for the pre-awareness and post-awareness groups

\begin{tabular}{|c|c|c|c|c|}
\hline & $\begin{array}{l}\text { Pre-awareness } \\
(n=150)\end{array}$ & $\begin{array}{l}\text { Post-awareness } \\
(n=150)\end{array}$ & Mean difference $(95 \% \mathrm{Cl})$ & $p$ value \\
\hline \multicolumn{5}{|l|}{ Adherence to key items (\%) } \\
\hline Unadjusted mean \pm SD & $46.1 \pm 15.5$ & $53.3 \pm 13.3$ & 7.3 (4.0 to 10.5$)$ & $<0.001^{\mathrm{a}}$ \\
\hline Adjusted EMM (95\% Cl) & $54.3(41.5$ to 67.1$)$ & $64.0(51.2$ to 76.9$)$ & 9.7 (6.9 to 12.5$)$ & $<0.001^{b}$ \\
\hline \multicolumn{5}{|l|}{ Time to recovery (days) } \\
\hline Unadjusted mean \pm SD & $4.8 \pm 2.4$ & $4.9 \pm 2.2$ & $0.0(-0.5$ to 0.6$)$ & $0.892^{\mathrm{a}}$ \\
\hline Adjusted EMM (95\% Cl) & $4.9(2.7$ to 7.0$)$ & 4.6 (2.5 to 6.8$)$ & $-0.2(-0.7$ to 0.2$)$ & $0.319^{b}$ \\
\hline \multicolumn{5}{|l|}{ Length of hospital stay (days) } \\
\hline Unadjusted mean \pm SD & $6.4 \pm 4.2$ & $6.5 \pm 4.1$ & $0.1(-0.9$ to 1.0$)$ & $0.874^{\mathrm{a}}$ \\
\hline Adjusted EMM (95\% Cl) & $6.6(2.6$ to 10.7$)$ & $6.3(2.2$ to 10.3$)$ & $-0.4(-1.3$ to 0.5$)$ & $0.419^{\mathrm{b}}$ \\
\hline
\end{tabular}




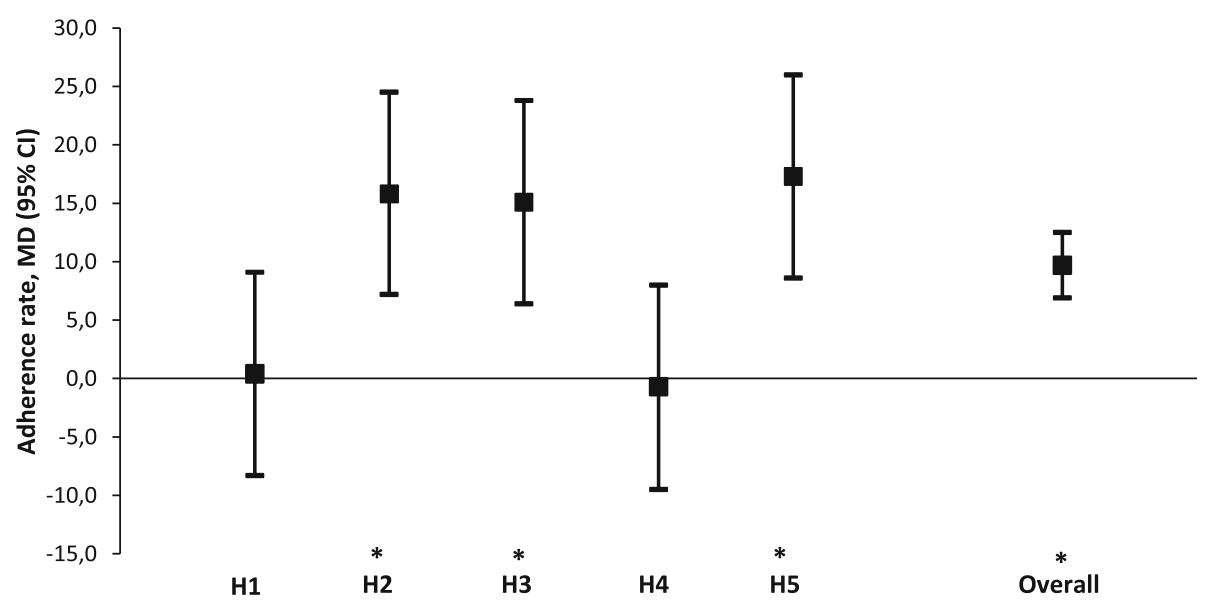

Fig. 1 Title: Mean differences in adherence rate to the preselected perioperative elements per hospital (pre-awareness versus post-awareness) Legend: Multivariable linear regression models were adjusted for age, ASA classification, diagnosis and type of surgery, histological subtype, surgical approach, duration of surgery, and type of additional surgical procedure. Hospital identifiers were entered in the models as dummy variables with interaction terms. ${ }^{*}$ indicates statistically significant difference. $\mathrm{Cl}=$ confidence interval, $\mathrm{MD}=$ mean difference

care. However, none of them reached the $70 \%$ cutoff and none managed to reduce the effect outcomes on time to functional recovery and length of hospital stay significantly. So, while there is some evidence that local stakeholder awareness of this intended change in perioperative practice might have affected the implementation of single evidence-based elements in nonparticipating hospitals, the improvement did not significantly or clinically change time to functional recovery or length of postoperative hospital stay. These findings are in agreement with Grol's theory regarding the several steps involved in the process of change [10]. Merely increasing awareness through external peers during recruitment activities does not seems sufficient in starting the cascade of change needed to implement complex, multimodal innovations.

Based on Rogers's diffusion theory [16], participating organizations probably belong to the early adopter and early majority categories. These organizations already have the best starting position for achieving beneficial implementation effects compared to nonparticipating organizations. The lack of clinical effect might be explained by differences in resources and willingness to change. Research on the effect of strategies used to implement innovations or guidelines should, therefore, not present data about the volunteering study sample only [27]. Additional information about nonparticipating organizations and contextual factors is particularly

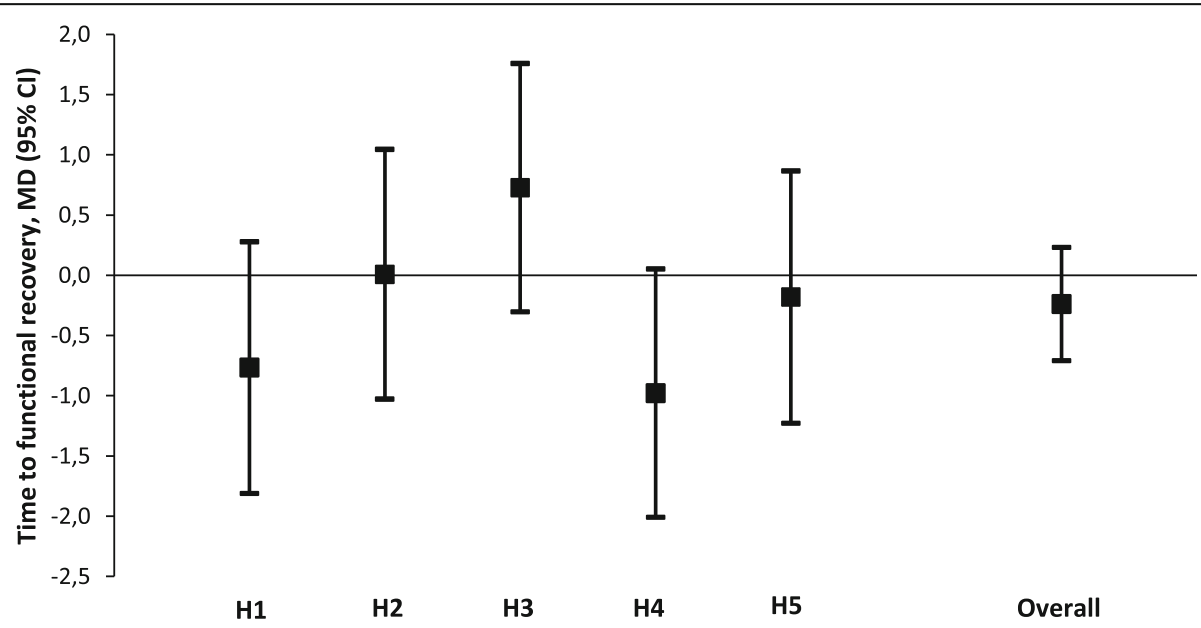

Fig. 2 Title: Mean differences in time to functional recovery (days) per hospital (pre-awareness versus post-awareness)Legend: Multivariable linear regression models were adjusted for age, ASA classification, diagnosis and type of surgery, histological subtype, surgical approach, duration of surgery, and type of additional surgical procedure. Hospital identifiers were entered in the models as dummy variables with interaction terms. Analysis of the log-transformed data showed similar results. No statistically significant differences were found. $\mathrm{Cl}=$ confidence interval, $\mathrm{MD}=$ mean difference 


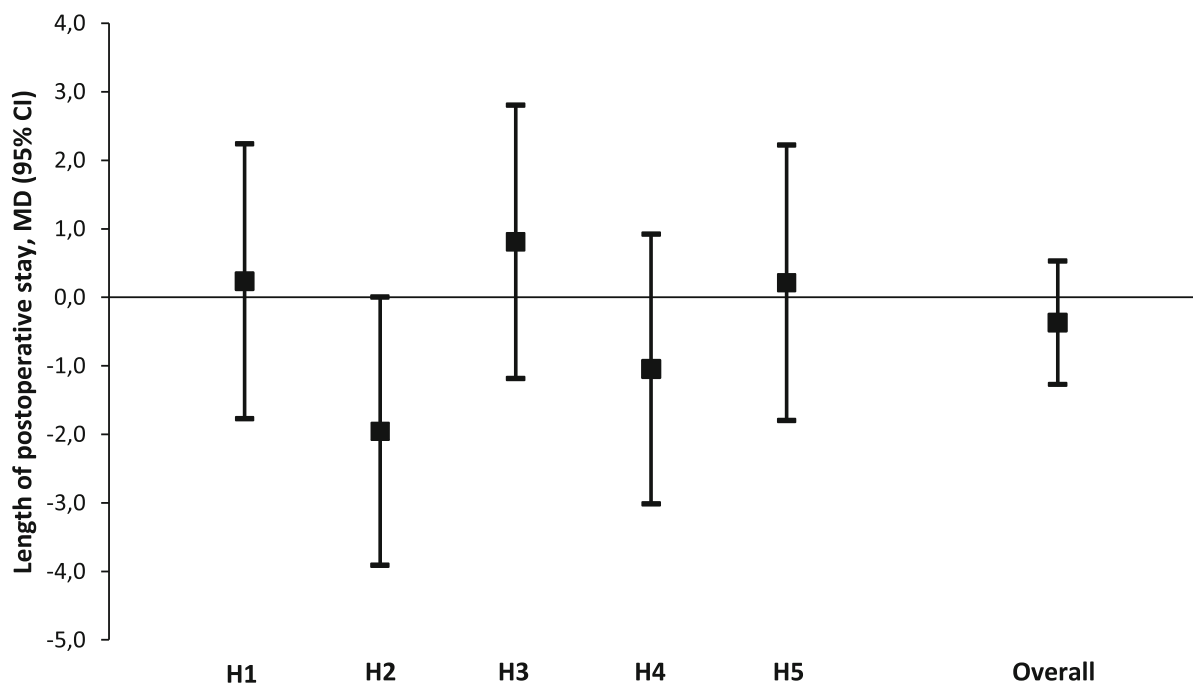

Fig. 3 Title: Mean differences in length of postoperative stay (days) per hospital (pre-awareness versus post-awareness)Legend: Multivariable linear regression models were adjusted for age, ASA classification, diagnosis and type of surgery, histological subtype, surgical approach, duration of surgery and type of additional surgical procedure. Hospital identifiers were entered in the models as dummy variables with interaction terms. Analysis of the log-transformed data showed similar results. No statistically significant differences were found. $\mathrm{Cl}=\mathrm{confidence} \mathrm{interval,}$

$\mathrm{MD}=$ mean difference

necessary to provide representative effect outcomes of different implementation strategies [27]. Mays et al. [28] showed that the public health organizations involved in research networks tend to be more engaged in implementation activities. Based on their findings, the authors suggest that the success of research networks is not only due to selection bias during the recruitment phase, but also to the development of competences during participation in research networks [28]. The findings of this quantitative study do not give insight into the underlying processes involved. Therefore, the limited effect could be due to the combination of a preexisting resistance to change and to a lack of resources and tools for achieving organizational change.

Organizational readiness seems to be essential in the process of change [11]. On the other hand, components of organizational readiness determine whether organizations become participants in implementation interventions [15]. In the beginning, we intended to include all eligible hospitals in the SINERGY trial. The process of recruiting hospitals was slow and difficult, despite the use of targeted strategies and facilitators. An evidence-based innovation that had already been proved effective, local stakeholders, peer pressure, and general funding of implementation strategies were used to increase participation. Eventually, a participation rate of $73 \%$ of all eligible hospitals was achieved. Stakeholders stated that their decision not to participate was based on organizational and financial constraints. This is in line with factors identified by a recent qualitative report [29]. In retrospect, because baseline outcomes in effect measures did not differ between participating and nonparticipating organizations they appear not to have influenced the choice to participate in implementation interventions. Prior studies have determined the importance of addressing local barriers to change during implementation interventions [9]. To increase organizational readiness and the extent of implementation effects, barriers for participating in large-scale implementation interventions also have to be taken into account. In addition, targeted implementation interventions based on specific organizational needs might be effective in increasing participation rates. Organizational readiness is difficult to quantify, therefore qualitative data could have provided more information. Besides, this could have helped to interpret quantitative findings.

Our findings give insight into the characteristics of and changes that took place in organizations that did not participate in a nationwide comparative effectiveness trial, but they must be interpreted with some caution. Despite the nationwide focus, only a relatively small number of hospitals could be included because of the specific study design. Multivariable regression analyses, with the hospital as a fixed instead of random factor, were used to explore the influence of awareness on perioperative care and outcomes. Therefore, the results are specific to our study sample and regression outcomes lack statistical generalizability. Regardless, we think that the perceived challenges of the recruitment phase and the observed trends in our study might be illustrative of other situations as well, particularly due to its nationwide focus. Data were retrieved from a consecutive sample of patients per hospital. The sample size of 30 
patients per hospital was mainly based on the volume norm for Dutch hospitals. To increase comparability between hospitals all patients included in the study underwent surgery within the same one-year period. Inclusion of a larger number of patients per hospital would have resulted in a longer study period, thereby increasing the chance of bias.

\section{Conclusions}

This multiphase observational study has shown that neither hospital characteristics nor perioperative outcomes at baseline differed, in retrospect, between the participating and nonparticipating departments of a national large-scale implementation project. Although a statistically significant improvement in evidence-based perioperative practice was achieved over time, the awareness raised by recruitment activities alone was not enough to facilitate change in functional recovery and length of hospital stay in the nonparticipating hospitals. Despite its exploratory nature, this study offers some insight into the influence of large-scale implementation projects on clinical practice in nonparticipating hospitals.

\section{Abbreviations \\ Cl: confidence intervals; DGOG: Dutch Gynecology Oncology Group; ERAS: enhanced recovery after surgery; MD: mean differences; SD: standard deviation; SINERGY: Stepped Implementation of Enhanced Recovery in Gynecology; ZonMw: the Netherlands Organization for Health Research and Development.}

\section{Acknowledgements}

The collaborating authors of the SINERGY nonparticipant group are: Willemien J. van Driel (Netherlands Cancer Institute, Amsterdam); Harm H. de Haan (Isala Klinieken, Zwolle); Dennis van Hamont (Amphia Hospital, Breda); Angela L.M. Kok (Amphia Hospital, Breda); Ward Hofhuis (Franciscus Hospital, Rotterdam); and Marc P.L.M. Snijders (Canisius Wilhelmina Hospital, Nijmegen).

\section{Funding}

The study was financially supported by the Netherlands Organization for Health Research and Development (ZonMw) (project number 837003002). The funders had no role in study design, data collection and analysis, decision to publish, or preparation of the manuscript.

\section{Availability of data and materials}

The datasets supporting the conclusions of this article may be available upon request. Contact jeanny.degroot@maastrichtuniversity.nl for further information.

\section{Authors' contributions \\ JG and MT conducted the study. JM, BW, CD, BS, and TW provided important guidance for the design and execution of the study. JG conducted the analyses and drafted the manuscript. MT, JM, BW, CD, BS, TW and all the group authors contributed to the interpretation of data and the revision of the manuscript. All authors read and approved the final manuscript and agreed to be accountable for all aspects of the work.}

\section{Ethics approval and consent to participate}

Since the study had an observational design and patient information was obtained anonymously, the Medical Ethical Committee of the Maastricht University exempted the study from further ethics review and informed consent (METC 14-5-083).

\section{Consent for publication}

Not applicable.

\section{Competing interests}

The authors declare that they have no competing interests.

\section{Publisher's Note}

Springer Nature remains neutral with regard to jurisdictional claims in published maps and institutional affiliations.

\section{Author details}

'Department of Family Medicine, CAPHRI, School for Public Health and Primary Care, Maastricht University, P.O. Box 616, 6200, MD, Maastricht, The Netherlands. ${ }^{2}$ Department of Obstetrics and Gynaecology, Maastricht University Medical Centre, P.O. Box 5800, 6202, AZ, Maastricht, The Netherlands. ${ }^{3}$ Department of Research, Netherlands Comprehensive Cancer Organisation (IKNL), P.O. Box 19079, 3501, DB, Utrecht, The Netherlands. ${ }^{4}$ Department of Quality and Safety, Maastricht University Medical Centre, P.O. Box 5800, 6202, AZ, Maastricht, The Netherlands. ${ }^{5}$ Department of Methodology and Statistics, CAPHRI, School for Public Health and Primary Care, Maastricht University, P.O. Box 616, 6200, MD, Maastricht, The Netherlands. ${ }^{6}$ Department of Clinical Epidemiology and Medical Technology Assessment, CAPHRI, School for Public Health and Primary Care, Maastricht University Medical Centre, P.O. Box 5800, 6202, AZ, Maastricht, The Netherlands. ${ }^{7}$ GROW - School for Oncology and Developmental Biology, Maastricht University Medical Centre, P.O. Box 5800, 6202, AZ, Maastricht, The Netherlands.

Received: 15 February 2018 Accepted: 19 December 2018

Published online: 29 December 2018

\section{References}

1. Grimshaw JM, Eccles MP, Lavis JN, Hill SJ, Squires JE. Knowledge translation of research findings. Implement Sci. 2012;7:50

2. Grimshaw JM, Thomas RE, MacLennan G, Fraser C, Ramsay CR, Vale L, et al. Effectiveness and efficiency of guideline dissemination and implementation strategies. Health Technol Assess. 2004;8(6):iii-v 1-72.

3. Gagliardi AR, Alhabib S, members of Guidelines International Network Implementation Working Group. Trends in guideline implementation: a scoping systematic review. Implement Sci. 2015;10:54.

4. Stetler CB, Mittman BS, Francis J. Overview of the VA Quality Enhancement Research Initiative (QUERI) and QUERI theme articles: QUERI series. Implement Sci. 2008;3:8.

5. Cochrane Effective Practice and Organisation of Care Review Group. Data collection checklist. Ontario: Institute of Population Health. University of Ottawa; 2011.

6. Schouten LM, Hulscher ME, van Everdingen JJ, Huijsman R, Grol RP. Evidence for the impact of quality improvement collaboratives: systematic review. BMJ. 2008:336(7659):1491-4.

7. Gagliardi AR. "More bang for the buck": exploring optimal approaches for guideline implementation through interviews with international developers. BMC Health Serv Res. 2012;12:404.

8. Solberg LI. If you've seen one quality improvement collaborative. Ann Fam Med. 2005;3(3):198-9.

9. Grol R, Wensing M. What drives change? Barriers to and incentives for achieving evidence-based practice. Med J Aust. 2004;180(Suppl 6):S57-60.

10. Grol R. Implementing guidelines in general practice care. Qual Health Care. 1992;1(3):184-91.

11. Weiner BJ, Amick H, Lee SY. Conceptualization and measurement of organizational readiness for change: a review of the literature in health services research and other fields. Med Care Res Rev. 2008;65(4):379-436.

12. Kringos DS, Sunol R, Wagner C, Mannion R, Michel P, Klazinga NS, et al. The influence of context on the effectiveness of hospital quality improvement strategies: a review of systematic reviews. BMC Health Serv Res. 2015;15:277.

13. Ovretveit J. Understanding the conditions for improvement: research to discover which context influences affect improvement success. BMJ Qual Saf. 2011;20(Suppl 1):i18-23.

14. Kilo CM. A framework for collaborative improvement: lessons from the Institute for Healthcare Improvement's breakthrough series. Qual Manag Health Care. 1998;6(4):1-13.

15. Wilson T, Berwick DM, Cleary PD. What do collaborative improvement projects do? Experience from seven countries. Jt Comm J Qual Saf. 2003; 29(2):85-93.

16. Rogers E. Diffusion of innovations. 5th ed. New York: Free Press; 2003. 
17. Maessen J, Dejong CH, Hausel J, Nygren J, Lassen K, Andersen J, et al. A protocol is not enough to implement an enhanced recovery programme for colorectal resection. Br J Surg. 2017;94(2):224-31.

18. de Groot JJ, Maessen JM, Slangen BF, Winkens B, Dirksen CD, van der Weijden T. A stepped strategy that aims at the nationwide implementation of the enhanced recovery after surgery programme in major gynaecological surgery: study protocol of a cluster randomized controlled trial. Implement Sci. 2015;10:106.

19. Barber EL, Van Le L. Enhanced recovery pathways in gynecology and gynecologic oncology. Obstet Gynecol Surv. 2015;70(12):780-92.

20. de Groot JJ, Ament SM, Maessen JM, Dejong CH, Kleijnen JM, Slangen BF. Enhanced recovery pathways in abdominal gynecologic surgery: a systematic review and meta-analysis. Acta Obstet Gynecol Scand. 2016;95(4):382-95.

21. Nelson G, Kalogera E, Dowdy SC. Enhanced recovery pathways in gynecologic oncology. Gynecol Oncol. 2014;135(3):586-94.

22. Ahmed J, Khan S, Gatt M, Kallam R, MacFie J. Compliance with enhanced recovery programmes in elective colorectal surgery. Br J Surg. 2010;97(5):754-8

23. Gustafsson UO, Hausel J, Thorell A, Ljungqvist O, Soop M, Nygren J, et al. Adherence to the enhanced recovery after surgery protocol and outcomes after colorectal cancer surgery. Arch Surg. 2011;146(5):571-7.

24. Nelson G, Altman AD, Nick A, Meyer LA, Ramirez PT, Achtari C, et al. Guidelines for pre- and intra-operative care in gynecologic/oncology surgery: enhanced recovery after surgery (ERAS ${ }^{\circ}$ ) society recommendations - part I. Gynecol Oncol. 2016;140(2):313-22.

25. Nelson G, Altman AD, Nick A, Meyer LA, Ramirez PT, Achtari C, et al. Guidelines for postoperative care in gynecologic/oncology surgery: enhanced recovery after surgery (ERAS ${ }^{\circ}$ ) society recommendations - part II. Gynecol Oncol. 2016;140(2):323-32.

26. Kehlet H. Multimodal approach to control postoperative pathophysiology and rehabilitation. Br J Anaesth. 1997;78(5):606-17.

27. Glasgow RE, Klesges LM, Dzewaltowski DA, Estabrooks PA, Vogt TM. Evaluating the impact of health promotion programs: using the RE-AIM framework to form summary measures for decision making involving complex issues. Health Educ Res. 2006;21 (5):688-94.

28. Mays GP, Hogg RA, Castellanos-Cruz DM, Hoover AG, Fowler LC. Public health research implementation and translation: evidence from practicebased research networks. Am J Prev Med. 2013:45(6):752-62.

29. Herbert G, Sutton E, Burden S, Lewis S, Thomas S, Ness A, et al. Healthcare professionals' views of the enhanced recovery after surgery programme: a qualitative investigation. BMC Health Serv Res. 2017;17(1):617.

Ready to submit your research? Choose BMC and benefit from:

- fast, convenient online submission

- thorough peer review by experienced researchers in your field

- rapid publication on acceptance

- support for research data, including large and complex data types

- gold Open Access which fosters wider collaboration and increased citations

- maximum visibility for your research: over $100 \mathrm{M}$ website views per year

At $\mathrm{BMC}$, research is always in progress.

Learn more biomedcentral.com/submissions 